\title{
Overview of neurodegenerative disorders associated with dementia: role of MRI
}

\begin{abstract}
Dementia is a syndrome leading to deterioration in cognitive function beyond what might be expected from normal ageing. The most common dementia syndromes include Alzheimer's disease (AD), frontotemporal lobe degeneration (FTLD) and vascular Dementia (VaD). Neuroimaging including CT and MRI have a key role in early diagnosis of neurodegenerative diseases. MRI is the corner stone modality and has superior role in detecting dementia associated abnormalities like atrophy of hippocampus, medial temporal lobe or that of parietal lobe. Vascular related changes including including white matter lesions, lacunar infarcts or strategic infarcts can also be better assessed on MRI. Early diagnosis of neurodegenerative diseases can lead to early start of therapy with improved clinical outcome.
\end{abstract}

Volume 3 Issue I - 2018

\author{
Amna Alvi,' Fatima Mubarak,' Asma Alvi' \\ 'Department of Radiology, Aga Khan University Hospital, \\ Pakistan \\ ${ }^{2}$ King Edward Medical University, Pakistan
}

Correspondence: Fatima Mubarak, Department of Radiology Aga Khan University Hospital Karachi, Pakistan,

Email fatima.mubarak@aku.edu

Received: August 27, 2017| Published: February 21, 2018

\section{Introduction}

World Health Organization describes dementia as a syndrome usually of a chronic or progressive nature in which there is deterioration in cognitive function (i.e. the ability to process thought) beyond what might be expected from normal ageing. It affects memory, thinking, orientation, comprehension, calculation, learning capacity, language, and judgement. ${ }^{1,2}$

Neuroimaging has a challenging role in early diagnosis of neurodegenerative diseases leading to dementia and to assess disease progression. MRI is the corner stone modality, multidetector CT can also play a role when MRI is contraindicated or when imaging is needed only to rule out surgically treatable etiology of cognitive impairment.

MRI protocol for dementia includes coronal-oblique T1-weighted images obtained by reformatting sagittal 3D T1 weighted sequence, axial T2- weighted images, axial FLAIR images and axial T2*weighted images. DWI is a supplemental sequence done in cases of rapidly progressive neuro degeneration especially in young patients. ${ }^{3,4}$

Typical MR findings in dementia includes atrophy of hippocampus, medial temporal lobe or that of parietal lobe or there can be global cortical atrophy. Other findings are vascular related including white matter lesions, lacunar infarcts or strategic infarcts (infarcts in areas which are involved in cognition). ${ }^{5}$

Hippocampal and medial temporal lobe atrophy is best assessed on coronal-oblique T1-weighted images. Axial T2-weighted images are used to assess infarctions especially thalamic and basal ganglia lacunar infarcts. Assessment of global cortical atrophy (GCA), vascular white matter hyperintensities and infarctions is done on axial FLAIR images. Axial T2*-weighted images are used to identify micro hemorhages in cerebral amyloid angiopathy and to see any calcification or iron deposition. ${ }^{6}$ The most common dementia syndromes include Alzheimer's disease (AD), frontotemporal lobe degeneration (FTLD) and vascular Dementia (VaD). ${ }^{7}$

Dementia can be the clinical manifestation of atypical Parkinson syndromes like dementia with Lewy bodies, progressive supranuclear palsy (PSP) and multi-system atrophy (MSA). MR brain including hippocampus is usually normal in cases of dementia secondary to
Lewy bodies deposition that differentiates it from AD. Significant atrophy of midbrain is seen in PSP giving the typical 'humming bird sign' on MRI. In MSA there is severe atrophy of cerebellum and pons. ${ }^{8}$ Cognitive impairment can also be seen in other conditions like cerebral amyloid angiopathy, Cerebral Autosomal Dominant Arteriopathy with Subcortical Infarcts and Leukoencehalopathy (CADASIL), Creutzfeldt-Jakob Disease (CJD) or in Huntington Disease. $^{9}$

$\mathrm{AD}$ is a gradually progressing disease with age as its major risk factor, affecting approximately $8 \%$ of individuals over the age of 65 and $30 \%$ over the age of 85 years. Medial temporal lobe atrophy (MTA) and parietal atrophy (particularly that of precuneus) is typically seen in this disease. In end-stage AD there is severe GCA. Presenile AD is one of its variant charcterised by significant parietal lobe atrophy particularly that of precuneus and posterior cingulum. Hippocampus is mildly affected or sometimes it is absolutely normal. ${ }^{10,11}$

$\mathrm{VaD}$ has relatively more sudden onset compared to $\mathrm{AD}$, usually associated with vascular risk factors, regarded as the second most common cause of dementia after AD. ${ }^{11}$ Global cortical atrophy (GCA), lacunar infarcts, white matter lesions (WML's) and 'strategic infarcts' are seen in VaD.

FTLD also known as Pick's disease, is a progressive disease, seen relatively more commonly in presenile age group. Pronounced frontal and/or temporal lobe atrophy is characteristic of FTLD. In one of its sub type's i-e Semantic Dementia, atrophy is strikingly asymmetrical mainly affecting left temporal lobe. ${ }^{13}$ GCA and WML's can also be the result of normal aging. ${ }^{14}$

The standardized systematic scoring of brain atrophy and that of vascular changes is essential while reading the MR images of patients presenting with dementia ${ }^{15-17}$ and it includes GCA-scale for Global Cortical Atrophy, Fazekas scale for white matter lesions, MTA-scale for Medial Temporal lobe Atrophy and Koedam score for Parietal Atrophy.

\section{Conclusion}

Delay in diagnosis of neurodegenerative disorders lead to worsening of symptoms and delay in treatment. Advancements in neuroimaging especially in MRI have made the early diagnosis 
possible that leads to early start of therapy with improved clinical outcome. MRI is the corner stone modality and has superior role in detecting dementia associated abnormalities like atrophy of hippocampus, medial temporal lobe or that of parietal lobe.

Brainimaging according to standard protocols forneurodegenerative disorders and scan interpretation by expert neuroradiologist has a major role in the management of these disorders.

\section{Acknowledgements}

None.

\section{Conflict of interest}

Authors declare that there is no conflict of interest.

\section{References}

1. http://www.who.int/mediacentre/factsheets/fs362/en

2. Dening T, Babu SM. Dementia: definitions and types. Nurs Stand. 2015;29(37):37-42.

3. Gasser MH. Clinical Neuroradiology, A Case Based Approach. UK; 2009. p. 103-114.

4. Osborne, Salzman, Barcovich. Diagnostic Imaging: Brain, 2nd edn. UK; 2012. p. $1-1266$

5. Flier V, Barkhof F, Verdelho A, et al. Small Vessel Disease and General Cognitive Function in Nondisabled Elderly. Stroke. 2005;36(10):2116-2120

6. António JBL, Elisabeth CW, Philip S, et al. Thalamic Lesions in Vascular Dementia. Low Sensitivity of Fluid-Attenuated Inversion Recovery (FLAIR) Imaging. Stroke. 2004;35:415-419.

7. CNS Degenerative Diseases. Webpath: the Internet Pathology Laboratory for Medical Education of the Florida State University College of Medicine, USA.
8. Barber R, Gholkar A, Scheltens P, et al. Medial temporal lobe atrophy on MRI in dementia with Lewy bodies. Neurology. 1999;52(6):1153-1158.

9. Collie DA, Sellar RJ, Zeidler M, et al. MRI of Creutzfeldt-Jakob disease: imaging features and recommended MRI protocol. Clin Radiol. 2001;56(9):726-739.

10. Petrella JR, Coleman RE, Doraiswamy PM. Murali Doraiswamy, Neuroimaging and Early Diagnosis of Alzheimer Disease: A Look to the Future. Radiology. 2003;226(2):315-336.

11. Inzitari D, Simoni M, Pracucci G, et al. Risk of rapid global functional decline in elderly patients with severe cerebral age-related white matter changes: the LADIS study. Arch Intern Med 2007;167(1):81-88.

12. Straaten EC, Scheltens P, Knol DL, et al. Operational definitions for the NINDS-AIREN criteria for vascular dementia: an interobserver study. Stroke; 2003;34(8):1907-1912.

13. Frisoni GB, Scheltens PH, Galluzzi S, et al. Neuroimaging tools to rate regional atrophy, subcortical cerebrovascular disease and regional cerebral blood flow and metabolism: consensus paper of the EADC. Journal of Neurology Neurosurgery and Psychiatry. 2003;74:1371-1381.

14. Wahlund LO, Barkhof F, Fazekas F, et al. New Rating Scale for AgeRelated White Matter Changes Applicable to MRI and CT on behalf of the European Task Force on Age-Related White Matter Changes. Stroke. 2001;32(6):1318-1322.

15. Vasconcellos LF, Pereira JS, Adachi M, et al. Correlation of MRI Visual Scales with Neuropsychological Profile in Mild Cognitive Impairment of Parkinson's Disease. Parkinson's Disease, Brazil; 2017. p. 1-7.

16. Koedam EL, Lehmann M, Flier WM, et al. Visual assessment of posterior atrophy development of a MRI rating scale. Eur Radiol. 2001;21(12):2618-2625

17. Inzitari D, Pracucci G, Poggesi A, et al. Changes in white matter as determinant of global functional decline in older independent outpatients: three year follow-up of LADIS (leukoaraiosis and disability) study cohort. LADIS Study Group. BMJ. 2009;6(339):b2477. 